\title{
The Immunomodulatory Activity of Mori folium, the Leaf of Morus alba L., in RAW 264.7 Macrophages In Vitro
}

\author{
Da Hye Kwonn', Ji Min Cheon ${ }^{2}$, Eun-Ok Choi ${ }^{1,3}$, Jin Woo Jeong ${ }^{1,3}$, Ki Won Lee ${ }^{4}$, Ki Young Kim4, Sung Goo Kim ${ }^{4}$, \\ Suhkmann $\mathrm{Kim}^{5}$, Su Hyun Hong ${ }^{3}$, Cheol Park ${ }^{6}$, Hye-Jin Hwang ${ }^{2}$, Yung Hyun Choi ${ }^{1,3}$ \\ ${ }^{1}$ Anti-Aging Research Center and ${ }^{2}$ Department of Food and Nutrition, Dongeui University, ${ }^{3}$ Department of Biochemistry, Dongeui University College \\ of Korean Medicine, ${ }^{4}$ Bio-Port Korea Inc., MarineBio-industry Development Center, ${ }^{5}$ Department of Chemistry, Pusan National University, \\ ${ }^{6}$ Department of Molecular Biology, Dongeui University, Busan, Korea
}

\begin{abstract}
Background: Immunoregulatory elements have emerged as useful immunotherapeutic agents against cancer. In traditional medicine, Mori folium, the leaf of Morus alba L. (Moraceae), has been used for various medicinal purposes; however, the immunomodulatory effects have not been fully identified. We evaluated the immunoenhancing potential of water extract of Mori folium (WEMF) in murine RAW264.7 macrophages.

Methods: RAW264.7 cells were treated with WEMF for 24 hours and cell viability was detected by an MTT method. Nitric oxide (NO) levels in the culture supernatants were assayed using Griess reagent. The productions of prostaglandin $E_{2}\left(P_{G} E_{2}\right)$ and immune-related cytokines was measured using ELISA detection kits. The mRNA and protein expression levels of Inducible NO synthase, COX-2, and cytokines were assayed by reverse transcription-PCR and Western blotting, respectively. The effect of WEMF on phagocytic activity was measured using a Phagocytosis Assay Kit.

Results: WEMF significantly stimulated the production of $\mathrm{NO}$ and $\mathrm{PGE}_{2}$ as immune response parameters at noncytotoxic concentrations, which was associated with the increased expression of inducible NO synthase and COX-2. The release and expression of cytokines, such as TNF- $\alpha$, interleukin (IL)-1 $\beta$, IL-6, and IL-10, were also significantly increased in response to treatment with WEMF. Moreover, WEMF promoted the macrophagic differentiation of RAW264.7 cells and the resulting phagocytosis activity.

Conclusions: WEMF has the potential to modulate the immune function by regulating immunological parameters. Further studies are needed to identify the active compounds and to support the use of WEMF as an immune stimulant.
\end{abstract}

(J Cancer Prev 2016;21:144-151)

Key Words: Mori folium, Macrophages, Immune response, Phagocytosis

\section{INTRODUCTION}

Among various immune system-related cells, macrophages are versatile cells that exist in almost all tissues and play important roles in immune responses. ${ }^{1,2}$ In particular, macrophages are recruited in infection sites where they are activated to perform many functions through increasing phagocytosis. This process is the first line of defense against microbial and parasitic infections and in removing senescent and dead cells, immune mediator secretion, and antigen presentation. ${ }^{3,4}$ Activated macrophages also prevent the invasion of pathogens by secreting inflammatory mediators, including nitric oxide $(\mathrm{NO})$, prostaglandin $\mathrm{E}_{2}\left(\mathrm{PGE}_{2}\right)$, and cytokines, such as TNF- $\alpha$ and interleukins (ILs). ${ }^{5-9}$

Recent data showed that natural compounds have been widely and safely consumed over centuries, and many studies have indicated that most natural compounds have a wide range of diverse biological activities but few side effects. ${ }^{10,11}$ These natural products could affect the development and progression of cancer

Received September 2, 2016, Revised September 4, 2016, Accepted September 4, 2016

Correspondence to: Yung Hyun Choi

Department of Biochemistry, Dongeui University College of Korean Medicine, 52-57 Yangjeong-ro, Busanjin-gu, Busan 47227, Korea Tel: +82-51-850-7413, Fax: +82-51-853-4036, E-mail: choiyh@deu.ac.kr, ORCID: Yung Hyun Choi, http://orcid.org/0000-0002-1454-3124

Copyright (C) 2016 Korean Society of Cancer Prevention

(c) This is an Open Access article distributed under the terms of the Creative Commons Attribution Non-Commercial License (http://creativecommons.org/licenses/by-nc/4.0) which permits unrestricted non-commercial use, distribution, and reproduction in any medium, provided the original work is properly cited. 
in various ways, such as inhibiting tumor cell growth, angiogenesis, and metastasis, immunomodulating, and enhancing effects of chemotherapeutic drugs. Therefore, traditional herbal medicinal resources have been investigated extensively for their immunomodulatory potential as adjuvants for therapeutic agents in immune-related functions. ${ }^{12-14}$ Morus alba L., white mulberry, is a deciduous tree that belongs to the Moraceae family, which is widely distributed in Asia. Mori folium, the leaf of $M$. alba L., has been used worldwide in traditional medicine from ancient times to the present for the treatment of various diseases. ${ }^{15,16}$ Previous studies have indicated that Mori folium possesses various pharmacological properties, including anti-microbial, ${ }^{17}$ antioxidant, ${ }^{18,19}$ anti-tumor, ${ }^{20}$ anti-obesity, ${ }^{21,22}$ anti-hypotensive, ${ }^{23}$ neuroprotective, ${ }^{24}$ and anti-diabetic actions. ${ }^{25}$ In addition, previous studies, as well as our recent data, ${ }^{26}$ indicated that the extracts and components of Mori folium had anti-inflammatory effects in different experimental models. ${ }^{2729}$ However, the effects and molecular mechanisms involved in such immunostimulatory actions have remained elusive. Therefore, in this study, as a part of our on-going research to find novel immunostimulatory active substances in traditional medicinal resources, we examined the immunostimulatory properties of water extract of Mori folium (WEMF) in RAW 264.7 mouse monocyte macrophages.

\section{MATERIALS AND METHODS}

\section{Preparation of water extract of Mori folium, reagents, and antibodies}

The dried leaves of $M$. albawere obtained from Bio-Port Korea (Busan, Korea), and WEMF was prepared as previously described. ${ }^{26}$ WEMF was dissolved in a $100 \mathrm{mg} / \mathrm{mL}$ concentration with distilled water, and the stock solution was then diluted with a culture medium to the desired concentration prior to use. Dulbecco's modified Eagle's medium (DMEM) and FBS were obtained from WelGENE (Daegu, Korea). Lipopolysaccharide (LPS, Escherichia coli serotype 055:B5), MTT, Griess reagent, and 4,6-diamidino-2-phenylindole were purchased from Sigma-Aldrich Chemical (St. Louis, MO, USA). Inducible NO synthase (iNOS), COX-2, TNF- $\alpha$, IL-1 $\beta$, IL-6, IL-10, and glyceraldehyde 3-phosphate dehydrogenase (GAPDH) oligonucleotide primers, and moloney-murine leukemia virus (M-MLV) reverse transcriptase kit were purchased from BioNEER (Daejeon, Korea). The ELISA detection kits for PGE 2 , TNF- $\alpha$, IL-1 $\beta$, IL-6, and IL-10 were obtained from R\&D Systems (Minneapolis, MN, USA). TRIzol reagent for the isolation of RNA was purchased from Invitrogen
Life Technologies (Carlsbad, CA, USA). The primary antibodies were purchased from Santa Cruz Biotechnology (Santa Cruz, CA, USA) and Cell Signaling Technology (Boston, MA, USA). Peroxidase-labeled donkey, anti-rabbit, and sheep anti-mouse immunoglobulin and an enhanced chemiluminescence (ECL) detection system were obtained from Amersham (Arlington Heights, IL, USA). All other chemicals were purchased from Sigma-Aldrich Chemical.

2. Cell culture, measurement of cell viability, and morphological analysis

The RAW 264.7 macrophage cell line was obtained from the Korean Cell Line Bank (Seoul, Korea) and cultured at $37^{\circ} \mathrm{C}$ in $5 \%$ $\mathrm{CO}_{2}$ containing DMEM supplemented by $10 \% \mathrm{FBS}, 100 \mathrm{U} / \mathrm{mL}$ of penicillin, and $100 \mathrm{mg} / \mathrm{mL}$ of streptomycin. Cell viability studies were performed using a colorimetric MTT assay. In brief, the RAW 264.7 cells were seeded at a density of $1 \times 10^{4}$ cells/well in a 96 well-plate and then incubated at $37^{\circ} \mathrm{C}$ for 24 hours. The cells were treated with various concentrations of WEMF or $0.5 \mathrm{ng} / \mathrm{mL}$ LPS for 24 hours. After the medium was discarded, an MTT solution (5 $\mathrm{mg} / \mathrm{mL}$ in PBS) was added to each well and incubated for another 3 hours at $37^{\circ} \mathrm{C}$. The medium was removed and dimethyl sulfoxide was added to dissolve the formazan dye. The optical density was read at $560 \mathrm{~nm}$ using a microplate spectrophotometer (Molecular Devices, Sunnyvale, CA, USA) to determine the cell viability. ${ }^{30}$ To assess the effect of WEMF on cellular morphology, the cells were photographed under an inverted microscope (Carl Zeiss, Oberkochen, Germany).

\section{Measurement of nitric oxide production in RAW 264.7 macrophages}

The accumulation of NO in the culture supernatants was assayed using Griess reagent. In brief, the cells were treated with various concentrations of WEMF or $0.5 \mathrm{ng} / \mathrm{mL}$ LPS for 24 hours. The supernatant was then collected and mixed with the same volume of Griess reagent for 10 minutes at room temperature in the dark. The absorbance was measured at $540 \mathrm{~nm}$ on a microplate reader, and NO concentrations were calculated by referencing a standard curve generated by known concentrations of sodium nitrite. ${ }^{31}$ A fresh culture medium was used as the blank in all experiments.

4. Measurement of prostaglandin $E_{2}, T N F-\alpha$, and interleukin-1 $\beta$ production in RAW 264.7 macrophages

To measure the production of $\mathrm{PGE}_{2}$, TNF- $\alpha$, IL-1 $\beta$, and IL-10, 
the cells were cultured under the same conditions as in the NO measurement assay. The levels of PGE 2 , TNF- $\alpha$, IL-1 $1 \beta$, and IL-10 in cultured media were determined by selective ELISA kits according to the manufacturer's instructions. ${ }^{32}$

\section{RNA isolation and reverse transcription-PCR}

Total RNA was isolated from the cultured cells using the TRIzol reagent according to the manufacturer's instructions and then reversely transcribed using the M-MLV reverse transcriptase kit to produce CDNAs. CDNAs were amplified by PCR using the desired primers. The PCR reaction was initiated at $94^{\circ} \mathrm{C}$ for 2 minutes, followed by 31 cycles at $94^{\circ} \mathrm{C}$ for 30 seconds, annealing temperature for 30 seconds, $72^{\circ} \mathrm{C}$ for 30 seconds, and the final extension step at $72^{\circ} \mathrm{C}$ for 5 minutes. Following amplification, the PCR products were separated by $1.5 \%$ agarose gel electrophoresis, stained with ethidium bromide, and visualized by ultraviolet illumination. In a parallel experiment, GAPDH was used as an internal control.

\section{Protein extraction and Western blot analysis}

The cell pellets were collected and resuspended in extraction lysis buffer ( $25 \mathrm{mM}$ Tris-Cl [pH 7.5], $250 \mathrm{mM} \mathrm{NaCl}, 5 \mathrm{mM}$ EDTA, $1 \%$ NP-40, $1 \mathrm{mM}$ phenymethylsulfonyl fluoride, and $5 \mathrm{mM}$ dithiothreitol) for 30 minutes at $4^{\circ} \mathrm{C}$. The protein concentration in the cell lysate was determined using a DC Protein Assay (Bio-Rad Laboratories, Hercules, CA, USA). Equal amounts of protein from each sample were separated by SDS-PAGE at $90 \mathrm{~V}$ for 2 hours and transferred onto polyvinylidene fluoride membranes (Millipore, Bedford, MA, USA). The membranes were then incubated overnight at $4^{\circ} \mathrm{C}$ with the corresponding primary antibodies, followed by incubation with the appropriate secondary antibodies conjugated to horseradish peroxidase at room temperature for 2 hours. An ECL detection system was used to monitor the immunoreactive bands.

\section{Phagocytosis assay}

The phagocytic ability of RAW 264.7 cells was detected using a Phagocytosis Assay Kit (IgG-FITC) (Cayman Chemical, Ann Arbor, MI, USA) according to the manufacturer's instructions. Briefly, RAW 264.7 cells were plated on a 4-well chamber slide and cultured overnight at $37^{\circ} \mathrm{C}$ to allow adherence to the plate. After treatment with WEMF for 24 hours, Latex Beads-Rabbit IgG-FITC Complex was added directly to the culture medium at a dilution of 1:200 and incubated at $37^{\circ} \mathrm{C}$ for 2 hours. The cells were then gently washed twice using a supplied assay buffer. After two washes with PBS, the cells were visualized at $\times 20$ magnification using a fluorescence microscope (Carl Zeiss). The extent of phagocytosis was determined by measuring the absorbance at $620 \mathrm{~nm}$.

\section{Statistical analysis}

All data are presented as mean \pm SD. Significant differences among groups were determined using the unpaired Student's $t$ test. A value of $P<0.05$ was accepted as statistically significant. All figures reflect the data obtained from at least three independent experiments.

\section{RESULTS}

\section{Water extract of Mori folium did not affect RAW 264.7 cell viability}

LPS, also known as lipoglycans and endotoxins, is present in the outer cell membrane of gram negative bacteria and can activate macrophages through the activation of cellular signaling

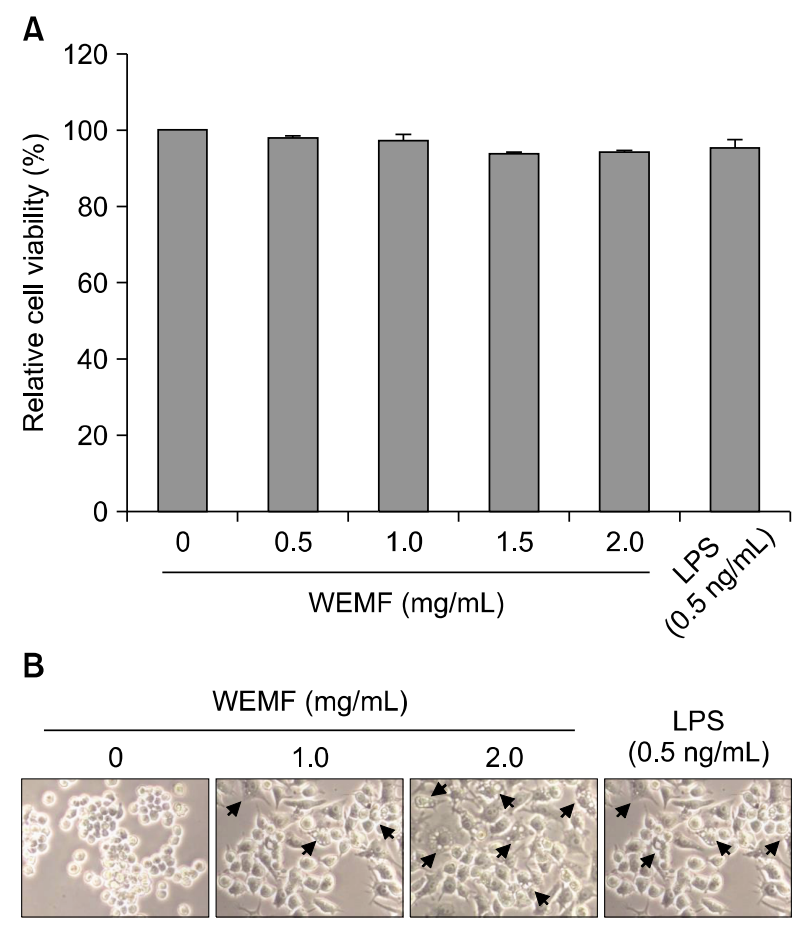

Figure 1. Effect of water extract of Mori folium on the viability and morphology of RAW 264.7 macrophages. The cells were treated with various concentrations of WEMF or $0.5 \mathrm{ng} / \mathrm{mL}$ LPS for 24 hours. (A) Cell viability was assessed with an MTT reduction assay, and the results were expressed as the mean $\pm \mathrm{SD}$ of three independent experiments. (B) Representative pictures of morphological changes of RAW 264.7 cells are shown. Arrows indicate activated macrophages $(200 \times$ original magnification). WEMF, water extract of Mori folium; LPS, lipopolysaccharide. 
pathways, thereby stimulating the transcription of genes, such as iNOS, COX-2, and macrophage-related cytokines. ${ }^{33,34}$ Thus, we used LPS as a positive substance to activate the macrophages. To determine the optimal concentration of WEMF, the RAW 264.7 cells were treated with various concentrations of WEMF for 24 hours. The MTT assay showed that up to $2.0 \mathrm{mg} / \mathrm{mL}$ WEMF and $0.5 \mathrm{ng} / \mathrm{mL}$ LPS were not cytotoxic (Fig. 1A). However, the WEMFand LPS-treated RAW 264.7 cells increased in size, and their surfaces became crude and rough, which indicated that WEMF might activate RAW 264.7 cells (Fig. 1B). We therefore selected 2.0 $\mathrm{mg} / \mathrm{mL}$ WEMF as the maximum concentration in further experiments with RAW 264.7 cells.

2. Water extract of Mori folium increased the production of nitric oxide and prostaglandin $E_{2}$ in RAW 264.7 macrophages

RAW 264.7 cells were treated with the indicated concentrations of WEMF for 24 hours in order to assess the effect of WEMF on the release of $\mathrm{NO}$ and $\mathrm{PGE}_{2}$. Following stimulation with WEMF, the levels of $\mathrm{NO}$ and $\mathrm{PGE}_{2}$ in the culture supernatants were determined by Griess reaction assay and ELISA, respectively. As

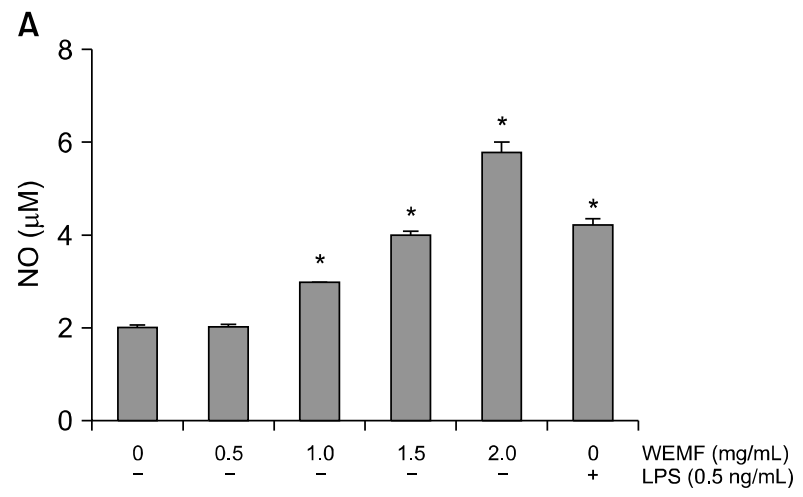

C

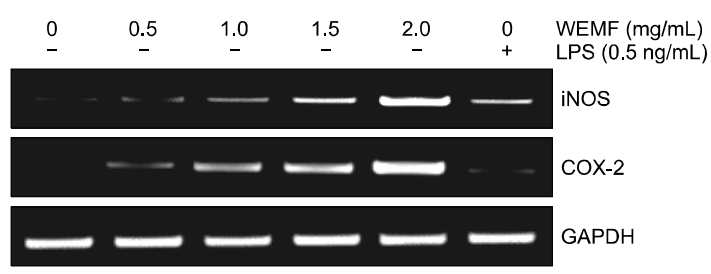

demonstrated in Figure 2A and 2B, the WEMF treatment significantly increased $\mathrm{NO}$ and $\mathrm{PGE}_{2}$ production compared to the unstimulated control cells. LPS, as a positive control, also induced the secretion of $\mathrm{NO}$ and of $\mathrm{PGE}_{2}$.

3. Water extract of Mori folium induced inducible nitric oxide synthase and COX-2 expressions at the mRNA and protein levels in RAW 264.7 macrophages

We subsequently investigated whether the inducible effects of WEMF on $\mathrm{NO}$ and $\mathrm{PGE}_{2}$ production were associated with mRNA and protein expression of their synthetic enzymes, iNOS and COX-2, by applying RT-PCR and Western blot analysis, respectively. As shown in Fig. $2 \mathrm{C}$ and 2D, WEMF effectively increased the mRNA and protein expression of iNOS and COX-2, as did LPS, suggesting that WEMF increased $\mathrm{NO}$ and $\mathrm{PGE}_{2}$ production by inducing the expression of their encoding genes.

\section{Water extract of Mori folium increased the secre- tion and expression of cytokines in RAW 264.7 macrophages}

To determine the effect of WEMF on the production of

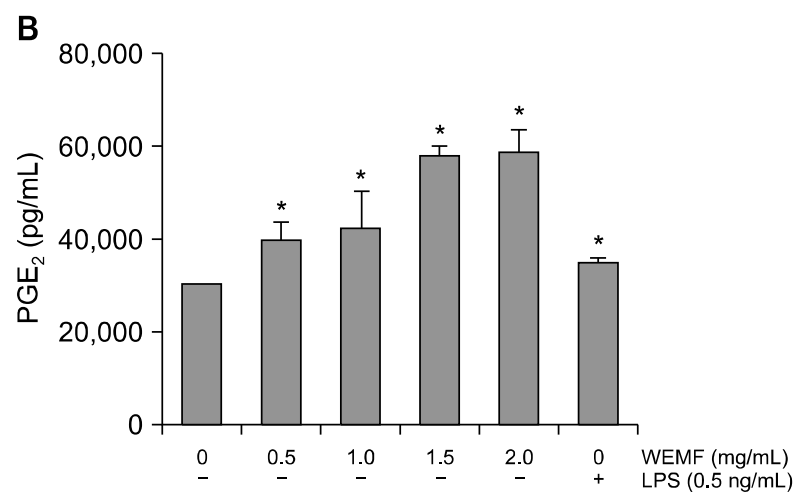

D

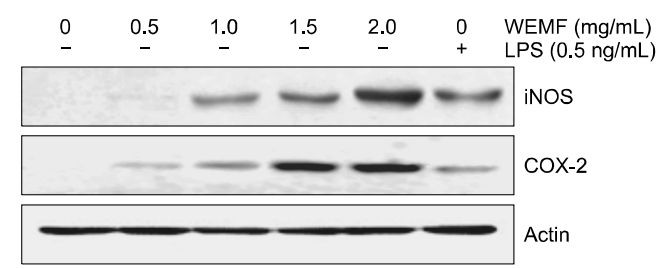

Figure 2. Induction of nitric oxide and prostaglandin $\mathrm{E}_{2}$ production by water extract of Mori folium in RAW 264.7 macrophages. The cells were treated with the indicated concentrations of water extract of Mori folium (WEMF) or $0.5 \mathrm{ng} / \mathrm{mL}$ LPS for 24 hours. The levels of (A) $\mathrm{NO}$ and (B) $\mathrm{PGE}_{2}$ in culture media were measured by Griess assay and a commercial ELISA kit, respectively. Each value indicates the mean \pm SD from three independent experiments $(* P<0.05$ compared to the control). (C) The total RNAs were prepared for RT-PCR analysis of the iNOS and COX-2 mRNA expression using the indicated primers in Materials and Methods. (D) Cell lysates were prepared for Western blot analysis with antibodies specific for murine iNOS and COX-2. The experiment was repeated three times and similar results were obtained. GAPDH and actin were used as the internal controls for the RT-PCR and Western blot analysis, respectively. NO, nitric oxide; PGE 2 , prostaglandin $\mathrm{E}_{2}$; WEMF, water extract of Mori folium; LPS, lipopolysaccharide; RT-PCR, reverse transcription-PCR; iNOS, inducible NO synthase; GAPDH, glyceraldehyde 3-phosphate dehydrogenase. 
cytokines of RAW 264.7 cells, the levels of cytokines in the culture media were measured by ELISA. As presented in Figure 3, the secretion of TNF- $\alpha$ and ILs (IL-1 $\beta$, IL-6, and IL-10) was significantly increased following WEMF and LPS treatment. The concentrations of TNF- $\alpha$ in the media of $2.0 \mathrm{mg} / \mathrm{mL}$ WEMF- and LPS-stimulated cells were approximately 9.5 and 7.5 times higher, respectively, than that of untreated control cells. Consistent with the results obtained from cytokines production, the mRNA and protein levels of TNF- $\alpha$ and ILs were also increased by WEMF treatment in a concentration-dependent manner (Fig. 4). These data indicate WEMF positively regulated the production of cytokines at the transcriptional and translational levels in RAW 264.7 cells.

\section{Water extract of Mori folium enhanced the pha- gocytic ability in RAW 264.7 macrophages}

Because phagocytosis is the primary function of macrophages, which leads to a diverse range of anti-microbial and cytotoxic responses, ${ }^{3,4}$ we examined the effects of WEMF on macrophage phagocytosis. As shown in Figure 5, the phagocytic properties of RAW 264.7 macrophages were dramatically increased by WEMF treatment.

\section{DISCUSSION}

Recently, the modulation of the immune response using natural products from herbal medicines has attracted interest as a possible therapeutic measure. ${ }^{3,35}$ Despite the diverse pharmacological potential of Mori folium, few studies have focused on its capacity to target immune effector cells. Therefore, we investigated whether WEMF possessed immune stimulatory potential in RAW 264.7 macrophages by examining its effects on the production of $\mathrm{NO}, \mathrm{PGE}_{2}$, and cytokines.

The excessive production of $\mathrm{NO}$ and $\mathrm{PGE}_{2}$, which are synthesized by their inducible enzymes, iNOS and COX-2, can kill normal cells and promote inflammation. However, at certain levels, they can be used as potential therapeutic tools and quantitative indexes of macrophage activation. ${ }^{7,36}$ In our study, WEMF treatment was found to stimulate the production of NO and $\mathrm{PGE}_{2}$ by promoting the expression of iNOS and COX-2 associated with morphological changes (Fig. 2), which demonstrated that WEMF is an effective activator of macrophages.
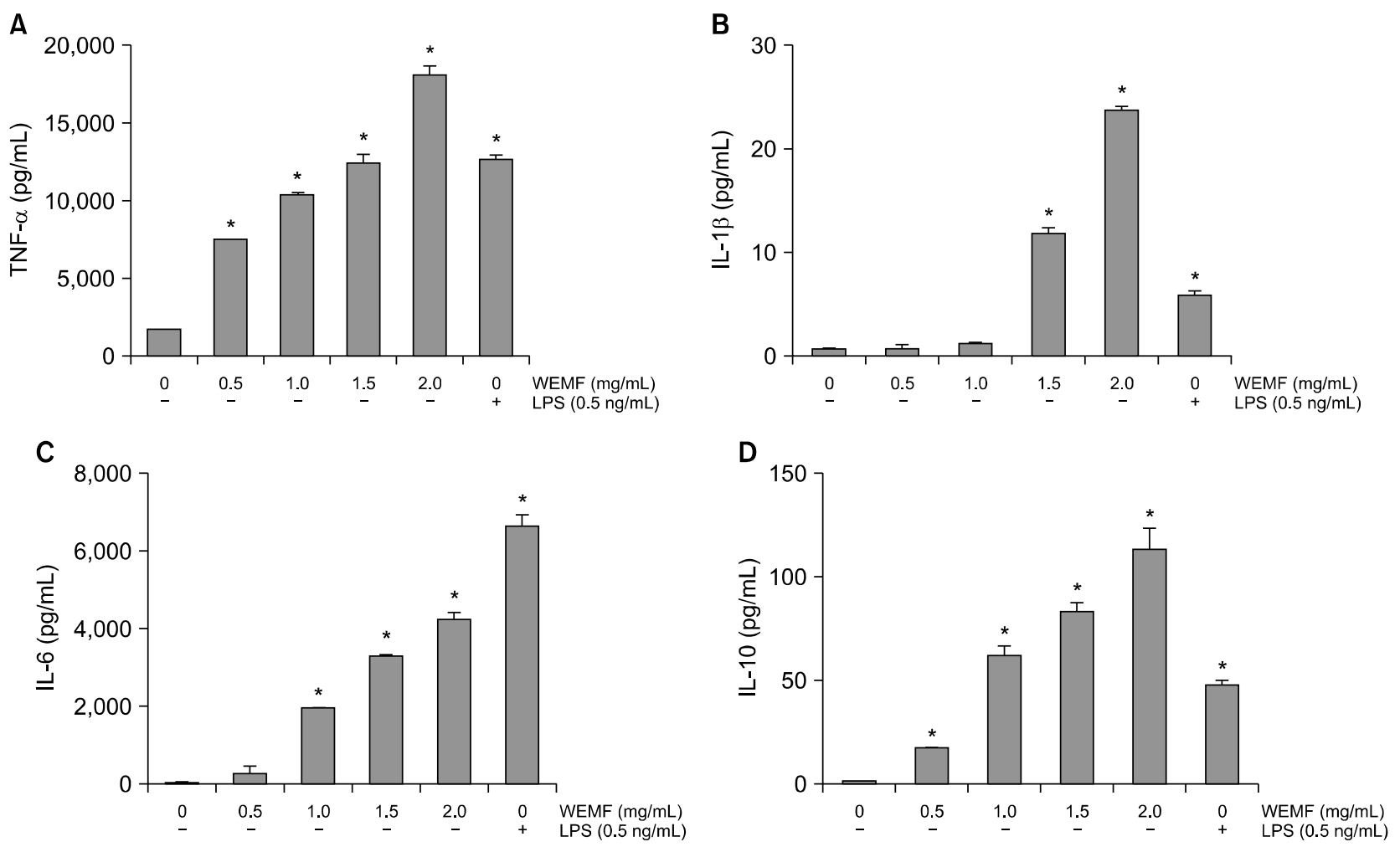

Figure 3. Increased secretion of cytokines by water extract of Mori folium in RAW 264.7 macrophages. The cells were treated with different concentrations of WEMF or $0.5 \mathrm{ng} / \mathrm{mL}$ LPS for 24 hours. The amounts of cytokines, such as (A) TNF- $\alpha$, (B) IL-1 $\beta$, (C) IL-6, and (D) IL-10, in the culture supernatants were measured by ELISA kits. WEMF, water extract of Mori folium; LPS, lipopolysaccharide; IL, interleukin. Data are presented as the means $\pm \mathrm{SD}$ of three independent experiments $(* P<0.05$ compared to the control). 
A

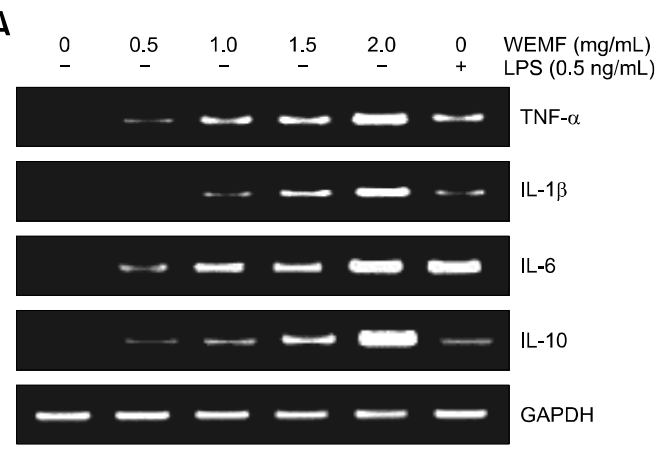

B

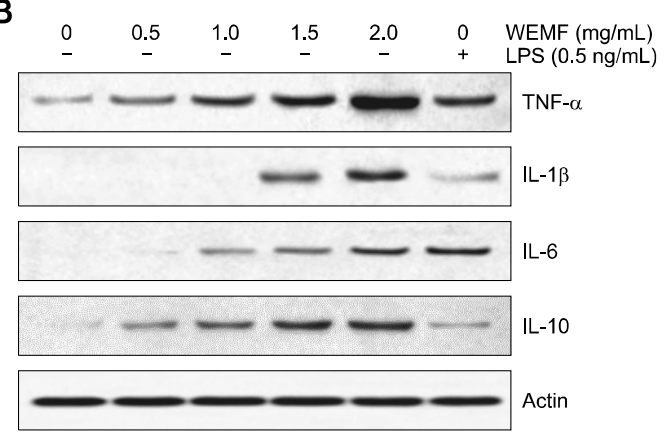

Figure 4. Effect of water extract of Mori folium on expression of cytokines in RAW 264.7 macrophages. (A) The cells were treated with different concentrations of WEMF or $0.5 \mathrm{ng} / \mathrm{mL}$ LPS for 24 hours. The total RNAs were isolated and RT-PCR was performed using the indicated primers in Materials and Methods. (B) Cell lysates were prepared for Western blot analysis with the specific antibodies against indicated cytokines. The experiment was repeated three times and similar results were obtained. GAPDH and actin were used as the internal controls for the RT-PCR and Western blot analysis, respectively. WEMF, water extract of Mori folium; LPS, lipopolysaccharide; RT-PCR, reverse transcription-PCR; GAPDH, glyceraldehyde 3-phosphate dehydrogenase; IL, interleukin.

Various immune-related cytokines, such as TNF- $\alpha$, IL-1 $\beta$, IL- 6 , and IL-10, are also mainly produced by activated macrophages for the modulation and orchestration of innate immunity. ${ }^{3.37}$ These cytokines can be produced from macrophages in response to bacterial LPS, infection, and inflammatory stimuli. They also play an important role in the immune system by aiding cytotoxic and cytostatic effects on infected or malignant cells. ${ }^{34,38}$ Among them, TNF- $\alpha$ is one of the earliest factors to be induced or activated in macrophages for eliciting tumor immunity. TNF- $\alpha$ plays as a key mediator of $\mathrm{T}$ lymphocyte and macrophage activation and exerts either beneficial or detrimental effects on mammalian cells by inducing the secretion of $\mathrm{NO}$ and $\mathrm{PGE}_{2}{ }^{6.39}$ Similarity, IL-1 $\beta$ and IL-6, and IL-10 are produced by various immune cells, including macrophages. These cytokines are essential for host survival of infection, and they are required for the repair of injured tissue. ${ }^{4,34}$ In our investigation, WEMF significantly stimulated the release and expression of these cytokines (Fig. 3 and 4), which indicates that it has an immunostimulatory effect on RAW 264.7 macrophages.

One of the most important roles of macrophages is phagocytosis, which comprises a variety of events that are mediated by the membrane receptors present on immune cells by binding to ligands on particle surfaces. ${ }^{40,41}$ Although phagocytosis is significantly involved in the acquisition of nutrients by some cells, it is also a crucial defense mechanism that provides protection against pathogen invasion and necrotic cellular debris scavenging in the immune system. ${ }^{3.4}$ Therefore, we further sought to determine whether WEMF increased phagocytic activity and found that WEMF significantly enhanced the capacity for phagocytosis in RAW 264.7 macrophages. The data suggested that WEMF, as a candidate immunostimulator in the innate immunity of macrophages (Fig. 5), might be used to defend against foreign particles, including bacteria, fungi, and unnecessary cellular debris.

According to our results, WEMF increased the production of immune modulators, such as $\mathrm{NO}$ and $\mathrm{PGE}_{2}$, by inducing their corresponding gene and protein expression in RAW 264.7 cells. WEMF also moderated the immune response by the increased release and expression of immune-related cytokines. Furthermore, WEMF enhanced the phagocytic activity of RAW 264.7 macrophages. Although further research is needed to identify the active pharmacological constituents of WEMF and to understand the mechanisms of their actions, these findings suggest that WEMF may be a useful immune stimulant. Further in vivo studies are needed to investigate in detail the overall immunostimulatory effects of WEMF.

\section{ACKNOWLEDGMENTS}

This research was a part of the project titled 'Omics based on fishery disease control technology development and industrialization,' funded by the Ministry of Oceans and Fisheries, Korea (20150242) and supported by the High Value-added Food Technology Development Program (314043-3), Ministry of Agriculture, Food and Rural Affairs, Republic of Korea.

\section{CONFLICTS OF INTEREST}

No potential conflicts of interest were disclosed. 
A

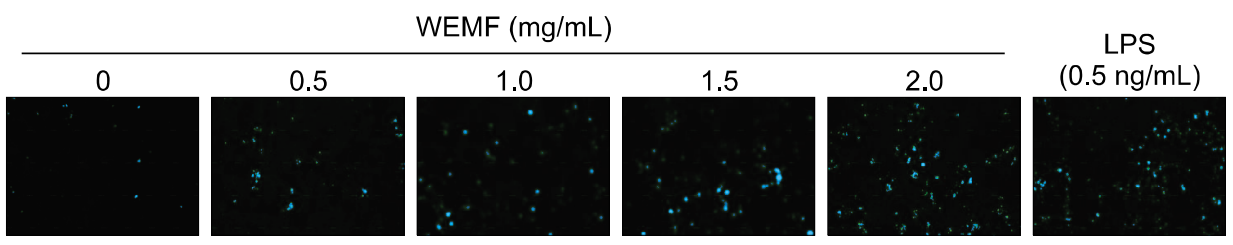

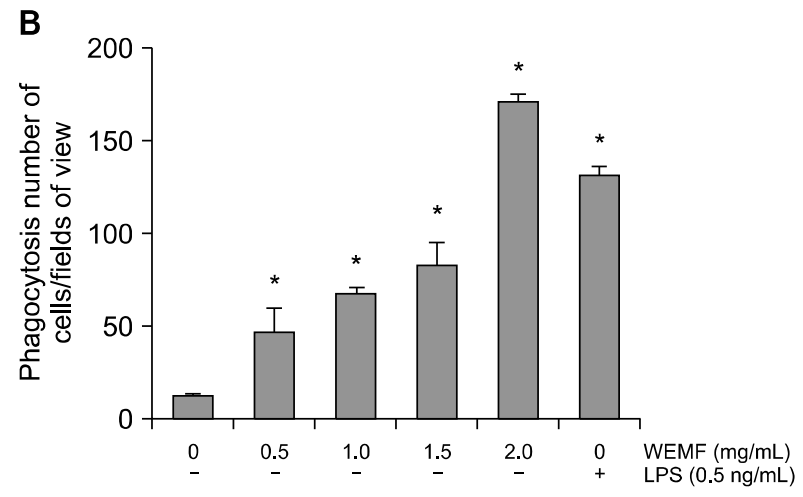

C

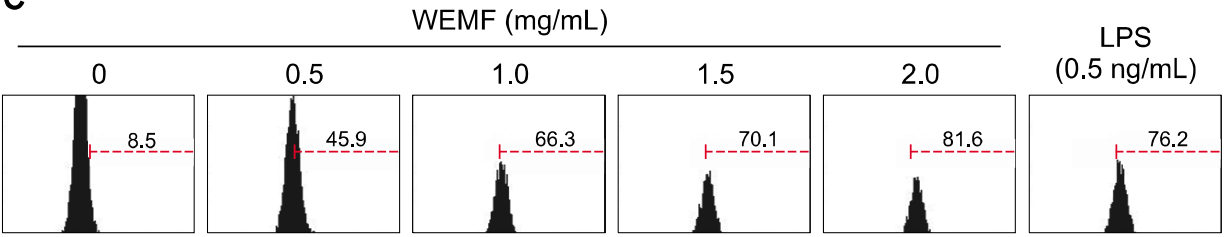

Figure 5. Effect of water extract of Mori folium on phagocytic activity in RAW 264.7 macrophages. Cells were treated with the indicated concentrations of WEMF or $0.5 \mathrm{ng} / \mathrm{mL}$ LPS for 24 hours. (A) The phagocytic cells were stained with Latex Beads-Rabbit IgG-FITC and visualized under a fluorescence microscope $(200 \times$ original magnification). (B) The number of phagocytic cells per field of view was counted and (C) the extent of phagocytosis was determined by measuring the absorbance at $620 \mathrm{~nm}$. WEMF, water extract of Mori folium; LPS, lipopolysaccharide. The data were presented as means \pm SD of three independent experiments $(* P<0.05$ compared to the control).

\section{REFERENCES}

1. Kudrin A, Ray D. Cunning factor: macrophage migration inhibitory factor as a redox-regulated target. Immunol Cell Biol 2008;86:232-8.

2. Krneta T, Gillgrass A, Ashkar AA. The influence of macrophages and the tumor microenvironment on natural killer cells. Curr Mol Med 2013;13:68-79.

3. Schultze JL, Schmidt SV. Molecular features of macrophage activation. Semin Immunol 2015;27:416-23.

4. McCabe A, MacNamara KC. Macrophages: key regulators of steady-state and demand-adapted hematopoiesis. Exp Hematol 2016;44:213-22.

5. Murakami A, Ohigashi H. Targeting NOX, INOS and COX-2 in inflammatory cells: chemoprevention using food phytochemicals. Int J Cancer 2007;121:2357-63.

6. Balkwill F. Tumour necrosis factor and cancer. Nat Rev Cancer 2009:9:361-71.

7. Kalinski P. Regulation of immune responses by prostaglandin E2. J Immunol 2012;188:21-8.

8. McNelis JC, Olefsky JM. Macrophages, immunity, and metabolic disease. Immunity 2014:41:36-48.

9. Decano JL, Mattson PC, Aikawa M. Macrophages in vascular inflammation: origins and functions. Curr Atheroscler Rep 2016;18:34.

10. Miyata T. Pharmacological basis of traditional medicines and health supplements as curatives. J Pharmacol Sci 2007;103:127-31.

11. Abuajah CI, Ogbonna AC, Osuji CM. Functional components and medicinal properties of food: a review. J Food Sci Technol 2015:52:2522-9.

12. Borchers AT, Hackman RM, Keen CL, Stern JS, Gershwin ME. Complementary medicine: a review of immunomodulatory effects of Chinese herbal medicines. Am J Clin Nutr 1997;66:1303-12.

13. Tan BK, Vanitha J. Immunomodulatory and antimicrobial effects of some traditional chinese medicinal herbs: a review. Curr Med Chem 2004:11:1423-30.

14. Ma HD, Deng YR, Tian Z, Lian ZX. Traditional Chinese medicine and immune regulation. Clin Rev Allergy Immunol 2013;44:229-41.

15. Yang Y, Tan YX, Chen RY, Kang J. The latest review on the polyphenols and their bioactivities of Chinese Morus plants. J Asian Nat Prod Res 2014;16:690-702.

16. Chan EW, Lye PY, Wong SK. Phytochemistry, pharmacology, and clinical trials of Morus alba. Chin J Nat Med 2016;14:17-30.

17. Tirupathi RG, Suresh BK, Ujwal KJ, Sujana P, Raoa AV, Sreedhar AS. Anti-microbial principles of selected remedial plants from Southern India. Asian Pac J Trop Biomed 2011;1:298-305.

18. Khan MA, Rahman AA, Islam S, Khandokhar P, Parvin S, Islam $\mathrm{MB}$, et al. A comparative study on the antioxidant activity of methanolic extracts from different parts of Morus alba L. (Moraceae). BMC Res Notes 2013;6:24.

19. Kim DS, Kang YM, Jin WY, Sung YY, Choi G, Kim HK. Antioxidant activities and polyphenol content of Morus alba leaf extracts col- 
lected from varying regions. Biomed Rep 2014:2:675-80.

20. Deepa M, Sureshkumar T, Satheeshkumar PK, Priya S. Antioxidant rich Morus alba leaf extract induces apoptosis in human colon and breast cancer cells by the downregulation of nitric oxide produced by inducible nitric oxide synthase. Nutr Cancer 2013;65: 305-10.

21. Sugimoto M, Arai H, Tamura $Y$, Murayama T, Khaengkhan $P$, Nishio T, et al. Mulberry leaf ameliorates the expression profile of adipocytokines by inhibiting oxidative stress in white adipose tissue in $\mathrm{db} / \mathrm{db}$ mice. Atherosclerosis 2009;204:388-94.

22. Ann JY, Eo H, Lim Y. Mulberry leaves (Morus alba L.) ameliorate obesity-induced hepatic lipogenesis, fibrosis, and oxidative stress in high-fat diet-fed mice. Genes Nutr 2015;10:46.

23. Kobayashi Y, Miyazawa M, Kamei A, Abe K, Kojima T. Ameliorative effects of mulberry (Morus alba L.) leaves on hyperlipidemia in rats fed a high-fat diet: induction of fatty acid oxidation, inhibition of lipogenesis, and suppression of oxidative stress. Biosci Biotechnol Biochem 2010;74:2385-95.

24. Xiang J, Tang YP, Zhou ZY, Wu P, Wang Z, Mori M, et al. Apocynum venetum leaf extract protects rat cortical neurons from injury induced by oxygen and glucose deprivation in vitro. Can J Physiol Pharmacol 2010;88:907-17.

25. Naowaboot J, Pannangpetch P, Kukongviriyapan V, Kukongviriyapan U, Nakmareong S, Itharat A. Mulberry leaf extract restores arterial pressure in streptozotocin-induced chronic diabetic rats. Nutr Res 2009:29:602-8.

26. Jeong JW, Lee HH, Lee KW, Kim KY, Kim SG, Hong SH, et al. Mori folium inhibits interleukin-1 $\beta$-induced expression of matrix metalloproteinases and inflammatory mediators by suppressing the activation of NF- $\mathrm{KB}$ and p38 MAPK in SW1353 human chondrocytes. Int J Mol Med 2016;37:452-60.

27. Hong $\mathrm{CH}$, Hur SK, Oh OJ, Kim SS, Nam KA, Lee SK. Evaluation of natural products on inhibition of inducible cyclooxygenase (COX-2) and nitric oxide synthase (iNOS) in cultured mouse macrophage cells. J Ethnopharmacol 2002;83:153-9.

28. Chao WW, Kuo YH, Li WC, Lin BF. The production of nitric oxide and prostaglandin E2 in peritoneal macrophages is inhibited by Andrographis paniculata, Angelica sinensis and Morus alba ethyl acetate fractions. J Ethnopharmacol 2009;122:68-75.

29. Shibata Y, Kume N, Arai H, Hayashida K, Inui-Hayashida A, Minami M, et al. Mulberry leaf aqueous fractions inhibit TNF-al- pha-induced nuclear factor kappaB (NF-kappaB) activation and lectin-like oxidized LDL receptor-1 (LOX-1) expression in vascular endothelial cells. Atherosclerosis 2007;193:20-7.

30. Oh K, Moon HG, Lee DS, Yoo YB. Tissue transglutaminase- interleukin-6 axis facilitates peritoneal tumor spreading and metastasis of human ovarian cancer cells. Lab Anim Res 2015;31: 188-97.

31. Lee H, Pyo MJ, Bae SK, Heo Y, Kim CG, Kang C, et al. Improved therapeutic profiles of PLA2-free bee venom prepared by ultrafiltration method. Toxicol Res 2015:31:33-40.

32. Wang L, Xu ML, Liu J, Wang Y, Hu JH, Wang MH. Sonchus asper extract inhibits LPS-induced oxidative stress and pro-inflammatory cytokine production in RAW264.7 macrophages. Nutr Res Pract 2015:9:579-85.

33. Ivashkiv LB. Inflammatory signaling in macrophages: transitions from acute to tolerant and alternative activation states. Eur J Immunol 2011:41:2477-81.

34. Morris MC, Gilliam EA, Li L. Innate immune programing by endotoxin and its pathological consequences. Front Immunol 2015:5:680.

35. Chen L, Yu J. Modulation of Toll-like receptor signaling in innate immunity by natural products. Int Immunopharmacol 2016;37:65-70.

36. Lorsbach RB, Murphy WJ, Lowenstein CJ, Snyder SH, Russell SW. Expression of the nitric oxide synthase gene in mouse macrophages activated for tumor cell killing. Molecular basis for the synergy between interferon-gamma and lipopolysaccharide. J Biol Chem 1993;268:1908-13.

37. Lavelle EC, McGuirk P, Mills KH. Molecules of infectious agents as immunomodulatory drugs. Curr Top Med Chem 2004:4:499-508.

38. Sherwin C, Fern R. Acute lipopolysaccharide-mediated injury in neonatal white matter glia: role of TNF-alpha, IL-1beta, and calcium. J Immunol 2005;175:155-61.

39. Vivier E, Raulet DH, Moretta A, Caligiuri MA, Zitvogel L, Lanier LL, et al. Innate or adaptive immunity? The example of natural killer cells. Science 2011;331:44-9.

40. Rosenberger CM, Finlay BB. Phagocyte sabotage: disruption of macrophage signalling by bacterial pathogens. Nat Rev Mol Cell Biol 2003;4:385-96.

41. Ostrowski PP, Grinstein S, Freeman SA. Diffusion barriers, mechanical forces, and the biophysics of phagocytosis. Dev Cell 2016;38:135-46. 